\title{
Evolutionary Changes in Bridge Designs
}

\author{
Dr Sakshi Madhok ${ }^{1}$, Dr Saksham Madhok ${ }^{2}$ \\ I( Asstt. Prof, Deptt of Prosthodontics, Aligarh Muslim University, India) \\ ${ }^{2}$ ( Post Graduate student, Deptt of Orthodontics, M.P.C.D\&R.C.Gwalior, India)
}

\begin{abstract}
Fixed Prosthodontics is a dynamic and ever evolving branch mainly because of the numerous advantages that it warrants over removable prosthesis. With the blooming implant technology fixed prosthesis has become very popular owing to its definite psychological advantage. As we all know the oral cavity is a difficult area to treat, many a times we encounter complicated clinical scenarios like periodontally weak abutments, non-parallel abutments, long edentulous spans, reduced crown - root ratio etc where fixed prosthodontic treatment option stands jeopardized. Over the years many innovative clinical designs based on modifications to the conventional bridges have been proposed by several authors so as to restore not only the appearance and function of the masticatory unit in such complicated and perplexing situations, but also the present and future health of the tissues. This review envisages the conventional bridge design to the most contemporary, as well as their variations that have been proposed by various authors which can be applied effectively in various clinical situations. These various designs till date, both of historical and clinical importance have been overviewed and presented forming a working classification of bridge designs.
\end{abstract}

Key words: Adhesive bridge, Andrew's bridge, Cantilever bridge, Rochette bridge, Virginia bridge,

\section{Introduction}

A fixed partial denture not designed abiding to the limitations of a given clinical situation could be disastrous. Every fixed partial denture is not limited to the most practical fixed- fixed bridge. Subtle modification in the design is needed taking into consideration the biomechanics and anatomical restrictions typical in every case and the patient's requirements. There are four basic conventional bridge designs that differ in the support provided at each end of the pontic . Other non-conventional designs are the result of innovative technology and an answer to a challenging clinical situation. A working classification of the bridge designs till date is as follows.

\section{Bridge Designs}

1. Conventional Bridges

1.1 Fixed-fixed

1.2 Fixed-movable

1.3 Cantilever

1.4 Spring cantilever

2. Resin retained

2.1 Bonded pontic

2.2 Rochette Bridge

2.3 Virginia Bridge

2.4 Maryland Bridge

2.5 Adhesive Bridge

3. Combination

3.1 Compound Bridge

3.2 Hybrid Bridge

4. Design Variations for special situations

4.1 Andrew's bridge

4.2 Removable Bridge

4.3 Telescopic Bridge

4.4 Looped connectors

5. Implant retained Fixed Prosthesis 


\section{Conventional Bridges.}

There are four basic conventional bridge designs that differ in the support provided at each end of the pontic.

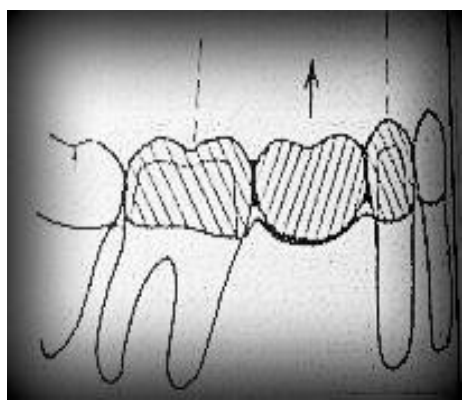

Figure 1

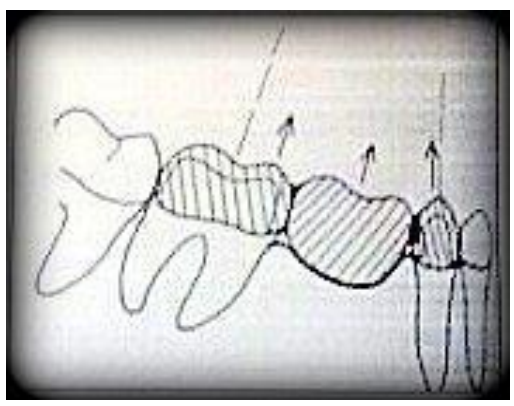

Figure 2

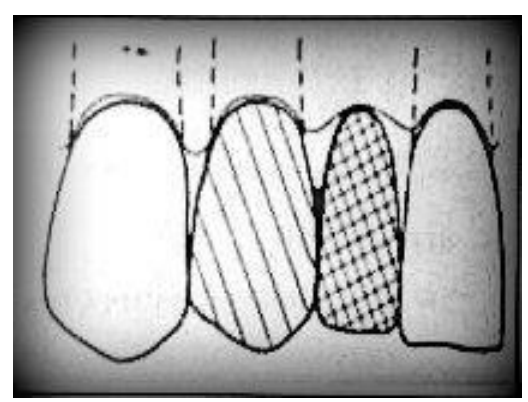

Figure3

\subsection{Fixed - fixed bridge}

The pontic rigidly connected to the abutments on both the sides is the conventional tooth supported fixed denture (Fig. 1). This design provides desirable strength and stability to the prosthesis. A fixed - fixed bridge should have all the occluding surface of the abutment teeth protected by the retainers otherwise an occlusal force directed at the unprotected area will depress the abutment tooth in its socket while the retainer is held by the bridge and abutment tooth. This will break down the cement bond causing leakage.

\subsection{Fixed - Movable bridge}

This form of bridge incorporates a stress distributing device which allows limited movement at one of the joints between the pontic and retainer (Fig. 2). The fixed end of the bridge has a rigid connector that is distal to the pontic. This is the major retainer which should cover the whole occluding surface of the tooth. The retainer carrying the movable joint is known as minor retainer and is not required to have a full occlusal coverage. A minor retainer can be as small as class II or class III inlay. This design is indicated in pier abutment and in cases of non - parallel abutment. It is contraindicated if the pier abutment is mobile or if the posterior abutment opposes an edentulous space or removable partial denture [1]. The "cantilever effect" of the non rigid design can place additional stress on the major retainer and therefore a strong abutment is required. Keyway is placed on the distal surface of the pier abutment while key on the mesial surface of the mesial pontic of the distal edentulous space. Nearly $98 \%$ of posterior teeth tilt mesially when subjected to occlusal forces [2]. If the key way is placed on the distal side, mesial movement seats the key into the keyway more firmly [3]. In the mandibular arch non rigid connectors are indicated when a complex fixed partial denture consists of anterior and posterior segments. Segmenting the complex mandibular fixed partial dentures can minimize the risk of FPD dislodgement due to mediolateral mandibular flexure during opening and closing stroke [4,5] Commonly used non-rigid connectors are - Tenon and Mortise connectors, looped connectors, cross pin and wing connectors, split pontic connectors and looped connectors.

\subsection{Cantilever bridge}

A very conservative design having pontic rigidly connected to a retainer at one end only ( Fig. 3). When the pontic is loaded occlusally the adjacent abutment tends to act as fulcrum with a lifting tendency on the farthest retainer[6]. To minimize the leverage effect the pontic should be kept as small as possible. There should be light occlusal contact with absolutely no contact in excursions. The pontic should possess maximum occlusogingival height to ensure a rigid prosthesis. To minimize the leverage imposed on the abutments they are often multiple [7], and the bridge is not used where the occlusal forces on the pontic will be heavy. If the root of abutment of a cantilever bridge is short or slender, rotation about its long axis may occur. This can be prevented by re-designing the pontic to provide some degree of wrap around the proximal aspect of unprepared tooth. Ideal cantilever situations are lateral incisor replaced with canine support and first premolar replaced with second premolar (primary abutment) and first molar (secondary abutment). It is a good option in case of nonparallel abutments however, it cannot be used in long span bridges.

\subsection{Spring Cantilever}

It is a tooth and tissue supported bridge. A pontic is supported at some distance from the retainer (Fig. 4). It is a type of cantilever bridge. Strong retention is required as for all cantilever bridges and double abutments are usually necessary. The retention of a spring bridge is severely tested when force is exerted in apicoincisal direction as seen on biting sticky food as the retainer is subjected to detrimental stresses. To avoid this, two retainers in adjacent teeth are used together to give added strength. This bridge design is used while replacing anteriors with diastema or in case of existing endodontically treated tooth posteriorly. This design 
cannot be used in lower arch because of lack of suitable tissue support. The bar should follow the natural contours of the rugae in the palate, so that it lies obscurely in the valleys and its

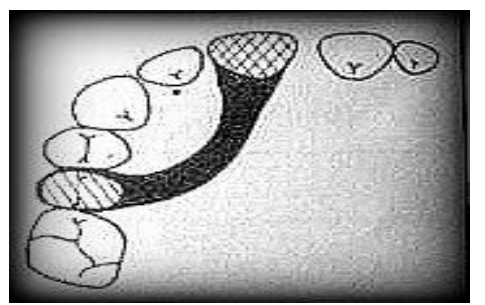

Figure 4

lateral margins do not represent an attraction to the tongue. The cross - section of the bar should be a flat, oval or a rounded - $\mathrm{T}$ shape[8]. The model should be lightly scraped to ensure firm seating on the soft tissue and minimize food trapping. High platinized gold or spring metal is used. Class IV casting gold is ideal. The Achilles heel with this design is the junction of retainer and the bar and the leverage on the abutment.

\section{Resin Retained Bridges}

These are minimal preparation bridges for resin retention luted to tooth structure, primarily enamel which has been etched to provide micromechanical retention for the resin cement. The principle is to cover as much enamel as possible without compromising occlusion, esthetics and periodontal health. It is minimally invasive bridge design which cannot be successful with our conventional cements. All four bridge designs discussed earlier are possible in resin retained bridges but only difference is that one requires retention with resin and the other with conventional frictional cements. This bridge can be given both in anterior and posterior segments provided the case selection is proper. Weakest link in resin retained prosthesis is the bond between the framework and resin. In the quest to improve the retention between resin and the metal framework various means have been developed - mechanical retention, micromechanical retention, macroscopic mechanical and chemical retention.

\subsection{Bonded Pontic.}

Introduced by Ibsen and Portnoy in 1973, these are the earliest resin retained prosthesis[9]. They are the resin tooth or patient's natural tooth bonded directly to the etched enamel. These are meant for short term replacements. The limiting factor was the weakness of the composite resin connector.

\subsection{Rochette Bridge.}

Resin retained FPD have gained popularity since the technique for splinting mandibular anterior teeth with the flared perforated metal casting was described by Rochette in 1973[10]. He used the technique principally for periodontal splinting and also used pontic in his design. It combined the advantage of resin bonding with the strength of cast metal retainer. This concept was later expanded to posterior teeth by Livaditis[11]. FABB Design utilizes perforated acrylic framework in place of metal framework[12]. Main problem with this design was a bulky framework, limited adhesion and exposed composite lute ( Fig 5).

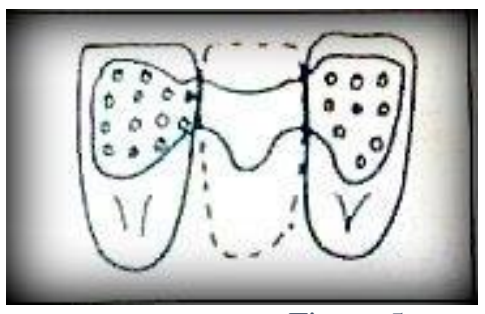

Figure 5

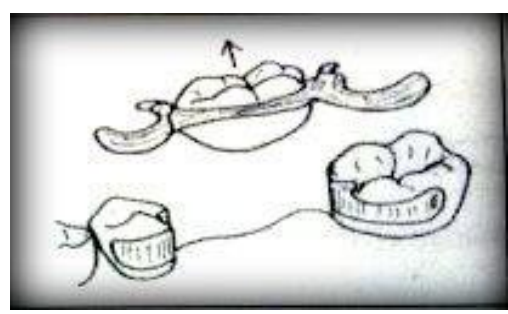

Figure 6

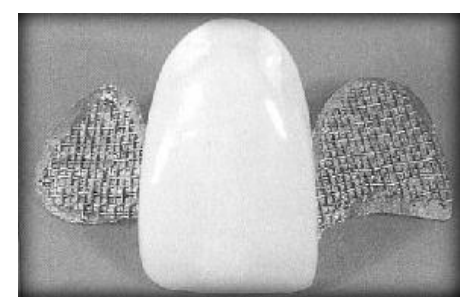

Figure 7

\subsection{Virginia Bridge}

It was first developed at Virginia Commonwealth University, School of Dentistry by Moon and Hudgins in 1984. It involves lost salt crystal technique giving the framework macroscopic mechanical means of retention with the resin cement $[13,14]$. On the working cast, within the outline of the retainer specially sized salt crystals $(150-250 \mu \mathrm{m})$ are sprinkled leaving $.5 \mathrm{~mm}$ border without crystals at the periphery of the pattern. Before investing the salt is dissolved from the pattern giving a rough surface for resin tag formation Retainers fabricated by this technique could be $30 \%-150 \%$ more retentive than retainers prepared by the electrochemical technique, depending on the resin used [15]. Another example of void containing framework is the technique involving cast mesh given by Shen in 1984 ( Fig 7). Example of net-like wire mesh used in cast 
mesh bridge is Kett- $\mathrm{O}$ - Bond ( Renfert) [1] . This technique gives moderate to good retention but the framework is bulky.

\subsection{Maryland Bridge}

Unlike Rochette bridge which utilizes mechanical means of retention the Maryland bridge incorporates micromechanical retention. Maryland bridges are resin bonded bridge using electrolytic etching of metal to retain the metal framework ( Fig 6). Thompson and Livaditis in 1983 developed a technique of electrolytic etching of $\mathrm{Ni}-\mathrm{Cr}$ and $\mathrm{Co}-\mathrm{Cr}$ alloy[16]. Etched cast retainers have definite advantage over cast perforated restorations. Retention is improved as resin to etched metal bond is substantially stronger than resin to etched enamel. In one study it was found that an electrochemically etched surface was approximately 2.9 times as retentive as a perforated one[17]. Retainers are thinner and non-perforated. Oral surface of cast retainers is highly polished and resists plaque accumulation. Whole surface area of retainer is retentive adding to retention. But etch is alloy specific requiring special apparatus. Only non-precious alloy which can be etched is used. Precious alloys cannot be etched. Micromechanical retention in noble alloys is achieved by electrolytic tin plating. Other means of micromechanical etching is Grit blasting using $50-250 \mu \mathrm{m}$ Aluminium Oxide. Chemical etching can be achieved by Hydrofluoric Acid gel ( Livaditis 1986) and Aqua Regia Gel [18].

\subsection{Adhesive Bridges}

Inspite of electrochemical etching being very popular in America, alloy etching and macroscopic retention is obsolete in Japan since 1980s. As a result of extensive research chemically active adhesive cements were developed for direct bonding to metal . These cements rely on chemical adhesion to the metal and not on microretention in the surface of the metal for bond strength. Etching was no longer necessary[19] Adhesive bridge shows chemical bonding between the metal and the resin luting agent. Direct bonding involves the chair side and lab systems. Metabond is first of these resin systems. It is based on formulation of Methylmetha acrylate (MMA) polymer powder and MMA liquid modified with adhesion promoter 4- META (4methacryloxyethyl trimellitate anhydride). Unique tributyl borane catalyst is added to liquid. On base metal alloys, Superbond has highest initial bond strengths of any adhesive resin systems[20]. But, it gives weak bond with high gold alloys and the bond shows hydrolytic instability. Introduction of Metabond was followed by Panavia which can be used both with high gold ( after tin plating) and base metal alloy. Tin plating can be done in lab, chair side or intraorally. Intraoral tin plating is done by tin amide solution. Adhesive monomer used in Panavia is MDP(10- methacrylolyloxydecyl dihydrogen phosphate). The phosphate end reacts with Calcium of tooth and with the metal oxide. Bond strength to etched base metal is greatly exceeded to that of tooth. Lab system for adhesive bonding have been developed. Silicoater Classical ( Tiller et. al, 1984) is based on the need for an intermediate layer containing silica as this provides sufficient bonding of the resin via a silane bonding agent. New version of Silicoater MD was introduced in 1998. This uses a special oven that burns a chrome endowing silica layer onto the surface. Pyrosil Pen Technology (1998) is the chairside version of silicoater. Rocatec System is a novel acrylic and metal bonding system which uses a tribochemical and thermal embedding of a silica layer by means of sand blasting on the metal surface[22]. Metal is thus rendered more reactive to resin via silane. It is unfortunate that this treatment modality is not very popular amongst dentist but if the case selection is proper it offers outstanding conservatism with tremendous bond strength.

\section{Combination}

\subsection{Compound Bridge}

A combination of any two or more of the conventional bridge designs is referred to as a compound bridge (Fig 8).

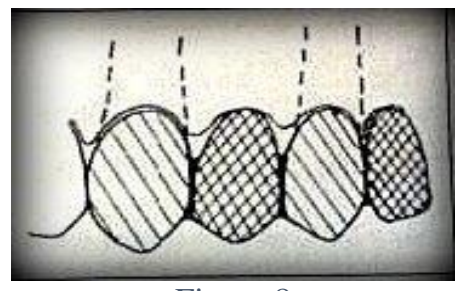

Figure 8 


\subsection{Hybrid Bridge}

Resin bonded retainers when used in combination with conventional retainers form hybrid bridges[8]. Fixed movable connectors are employed in case of hybrid bridges. Resin bonded retainer acts as the major retainer so that debonding does not require replacement of conventional retainer. It is also easier to create room for a movable joint within the confines of a conventional retainer.

\subsection{Andrew's Bridge}

\section{Design Variations For Special Situations}

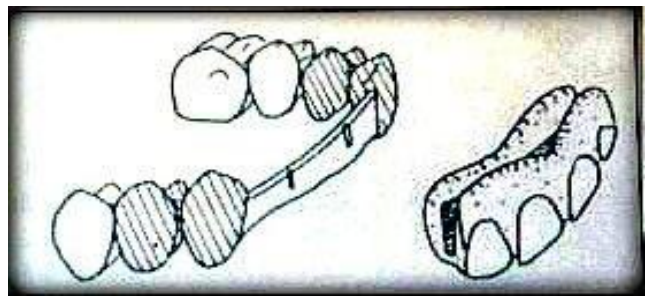

Figure 9.

It is a fixed RPD having a fixed and a removable component. The fixed part has a bar connected to two retainers on either side of the edentulous space and harbors a removable acrylic partial denture (Fig 9). The bar should be rectangular with parallel sides, height more than the width[23]. This bridge is indicated in edentulous ridges with severe vertical deficit especially in anteriors and cannot be used in reduced interarch space. One of the first attempts of fixed RPD was by Fossume in 1904, who used a round bar attached to abutment crowns that supported a suprastructure. Rotational stability was provided by flanges covering soft tissue. In 1905 Bennet came up with Bennet blade using a precision or semi-precision bar and sleeve assembly that provided rotational stability to be gained directly through substructures and abutment teeth. Variations in bar and clip concepts were developed by Dolder, Hader and Baker. These concepts relied on extended tissue coverage for rotational stability. In 1966 Andrew came up with Andrew's bridge system. It consists of prefabricated parallel rectangular specially designed bars and a matching sleeve to which replaced teeth and other supra-structure elements were attached. Unlike other bar systems, these prefabricated units were made of precision machined stainless steel rather than gold alloy. Very high tensile and yield strengths were claimed for the material so that the bar could be made thin and also occupy minimal vertical space. Two types of bars were manufactured: a single bar to use anteriorly and a twin bar for posterior gaps. These bars were available in three lengths of three different curvatures. Each curve was a segment of a circle and the combinations allowed adaptation to most clinical situations. Since the bar formed part of the arc of a circle, it simplified reconstruction should a patient lose or damage removable sections. This bridge design gives very good retention and stability with optimum esthetics.

\subsection{Removable Bridge}

It is a kind of telescopic prosthesis, with its first written reference in 1883 by Dexter. It is a periodontal prosthesis. These differ from the traditional bridges. It has a provision for modification and conversion in case of future loss of abutment teeth[24]. This is used when preservation of abutments is desired with a stable less retentive prosthesis than with a more retentive rigid prosthesis for a shorter period of time. With large bridges there are disadvantages in permanent cementation in that the maintenance and further endodontic or periodontal treatment of abutment teeth is difficult and if something goes wrong with one part of the bridge or abutment usually the whole bridge has to be sacrificed. For this reason larger bridges including full arch bridges are sometimes made so that they can be removed by the dentist without being destroyed. This is done by cementing individual cast gold copings to each of the abutment teeth, followed by temporary cementation of a superstructure bridge on the copings. Precision milling machines can be used for this. It is used in periodontally weak teeth, cariously involved teeth, endodontically treated teeth, non-parallel abutments and long edentulous span. It allows for easy removal of bridge so as to make necessary changes, repairs, extractions, pontic conversions etc. The disadvantage with this design is extensive lab work, limited retention and stability and that it requires tooth preparation. 
5.3 Telescopic Prosthesis

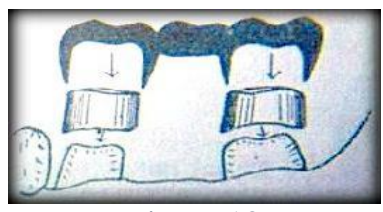

Figure 10

The retainer is constructed in two parts, an inner open topped sleeve made of hard gold and an outer full crown to cover both abutment crowns and inner sleeve ( Fig 10). The use of telescopic crown enables the mesial and distal surfaces to be prepared for one line of insertion (the inner sleeve) while the line of insertion of the bridge is reproduced on the outer surface of the sleeve. This design is useful in non parallel abutments and involves a comparatively less conservative tooth preparation.

\subsection{Looped connectors.}

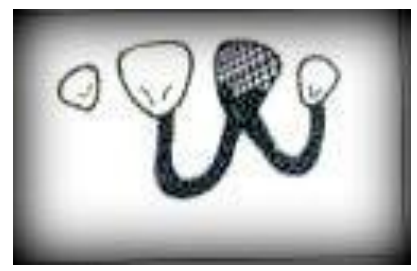

Figure 11

These are used to maintain esthetics in extreme diastema cases where conventional bridges cannot help. It interferes with the tongue movement and also with occlusion in some patients ( Fig 11).

\section{Implant Retained Fixed Prosthesis}

Introduction of implants in dentistry has opened up a myriad of treatment options. It could be in the form of single tooth implants or implant acting as abutments in long span bridges. Initial concept of bone driven implant placement is replaced by restoration driven implant placement ( Garbar 1993). This concept mandates that implant be placed where one can achieve maximum functional and esthetic benefits. If the desired site is lacking in bone or soft tissue augmentation procedures may be employed. Implants may replace partial or total dentition and may be screw or cement retained. It is being used to replace the crown (FP1), a portion of root (FP2) and even a portion of edentulous site (FP3). Implant retained FPDs are ideally suited for use where there are insufficient numbers of abutment or inadequate strength in abutments to support conventional FPD.

\section{Summary}

A common axiom in conventional prosthodontics for partial edentulism is a fixed partial denture. Fewer the natural teeth missing better the indication for fixed partial denture. Unfortunately every case is different in relation to anatomical variations, patient's desires and medical condition. There is no iron clad rule for a particular design. Subtle modifications in designing is required to suit a given particular case. Dentistry is an ever changing branch. Over the period of time many types of bridges have come up ranging from the traditional to resin retained to the implant retained bridges. With the treatment options available today, a dentist's knowledge and vigilance, adequate skilled lab support and patient's cooperation can help achieve the only goal of a prosthodontist that is meticulous replacement with maximum preservation.

\section{References}

[1]. Schillinburg H.T. et al. Fundamentals of fixed prosthodontics (Chicago, Quintessence, Third edition)

[2]. Picton DCA. Tilting movements of teeth during biting. Arch Oral Biol 1962;7:151-159

[3]. Schillinburg H.T, Fisher D.W. Non-rigid connectors for fixed partial dentures. J Am Dent 1973;87:1195 -1199.

[4]. Goodkind R.J, Heringlake C.B. Mandibular flexure in opening and closing movements, J Prosthet Dent 1973; $30: 134$

[5]. Fischman BM. The influence of fixed splints on mandibular flexure. J Prosthet Dent 1976; $35: 643$

[6]. Schweitzer JM, Schweitzer RD, Schweitzer J. Free-end pontics used on fixed partial dentures. J Prosthet Dent 1968;20:120138.

[7]. Wright WE. Success with the cantilever Fixed partial denture. J Prosthet Dent 1986; 55: 537-539

[8]. Allan DN, Foremen PC. Crown and Bridge Prosthodontics : an illustrated handbook (Briston, Wright1986)

[9]. Ibsen RL. One appointment technique using an adhesive composite. Dent Survey 1973;49:30-32 
[10]. Rochette AL. Attachment of a splint to enamel of lower anterior teeth. J Prosthet Dent 1973;30:418-423.

[11]. Livaditis GJ. Cast metal resin bonded retainers for posterior teeth. J Am Dent Assoc 1980;110:926-929

[12]. McLaughlin G. Direct bonded retainers- The advanced alternative (Philladelphia, Lippincott)

[13]. Hudgins JL, MoonPC, Knap FJ. Particle roughened resin bonded retainers. J Prosthet Dent 1985;53:471-476

[14]. Moon PC. Bond strengths of the lost salt procedure: A new retention method for resin bonded fixed prosthesis. J Prosthet dent 1987;57:435-439

[15]. El- Sherif MH, El- Messery A, Halhoul MN: The effects of alloy surface treatments and resins on the retention of resinbonded retainers. J Prosthet dent 1991; 65:782-786.

[16]. Livaditis J, Thompson VP. Etched castings: An improved retentive mechanism for resin bonded retainers. J Prosthet dent 1982;47:52-58

[17]. Dhillon M, Fenton AH, Watson PA: Bond strengths of composite to perforated and etched metal surfaces. J Dent Res $1983 ; 62: 304$.

[18]. Doukoudakis S, Cohen B, Tsoutsos. A new chemical method of etching metal frameworks of the acid etched prosthesis. J Prosthet Dent 1987; 58:421-423

[19]. Barrack WS, Lacy AM: A clinical technique for bonding gold castings to teeth. Quintessence Int 1993; 24: $701-713$.

[20]. Rosensteil SF et. al. Contemporary Fixed Prosthodontics( St Louis, Mosby, Third edition)

[21]. Eakle WS, Lacy AM. A clinical technique for bonding gold castings to teeth. Quintessence Int 1991; 22: 491-494.

[22]. Guggenberger R. Rocatec System- Adhesion by tribochemical coating. Dtsch Zahnarztl Z. 1989;44(11):874-6.

[23]. Kantorowicz GF, Howe et.al. Crowns and bridges( Butterworth Heinmann, Oxford, $5^{\text {th }}$ edition)

[24]. Smith BGN. Dental crowns and bridges: Dsesign and preparation ( London, Martin Dunitz) 